\title{
Subcutaneous Adipose Tissue Segmentation in Whole-Body MRI of Children*
}

\author{
Geoffroy Fouquier ${ }^{1}$, Jérémie Anquez ${ }^{1}$, Isabelle Bloch ${ }^{1}$, \\ Céline Falip ${ }^{2}$, and Catherine Adamsbaum ${ }^{2,3}$ \\ 1 Telecom ParisTech, CNRS LTCI, and Whist Lab, Paris, France \\ 2 Service de radiologie pédiatrique, Hôpital Saint Vincent de Paul, Paris, France \\ 3 Université Paris Descartes, faculté de médecine, Paris, France
}

\begin{abstract}
In this paper, we propose a new method to segment the subcutaneous adipose tissue (SAT) in whole-body (WB) magnetic resonance images of children. The method is based on an automated learning of radiometric characteristics, which is adaptive for each individual case, a decomposition of the body according to its main parts, and a minimal surface approach. The method aims at contributing to the creation of WB anatomical models of children, for applications such as numerical dosimetry simulations or medical applications such as obesity follow-up. Promising results are obtained on data from 20 children at various ages. Segmentations are validated with 4 manual segmentations.
\end{abstract}

\section{Introduction}

This paper is a first step towards the development of semi-automatic approaches for WB magnetic resonance images (MRI) segmentation and designing 3D models of the anatomy of children. These models are meant for dosimetry studies, to assess the children exposure to electromagnetic fields. We propose to build a set of models representing children at different ages, and suitable for deformations in order to test different postures during dosimetry studies, and hence generating a larger set of models from an existing set.

Our purpose is thus the realistic modeling of the human body in a semiautomatic way, since manual processing of the data is time consuming and cannot ensure to obtain topologically correct and smooth structures due to slice by slice processing. The process relies first on identifying the main body subparts by analyzing the body silhouette. Then, we propose to decompose the subject anatomy by segmenting SAT, the muscles and the bones, which represent about $80 \%$ of the subject body mass. In this article we describe the first step of this approach and SAT segmentation. In the context of realistic modeling, accuracy is not the main expected feature of the method since the segmentation is not aimed at providing an exact individual model of each patient. What is important is that the fat is well located, topologically correct, and approximately fits the

\footnotetext{
* This work was partially supported by the French National Research Agency (ANR) within the KidPocket project. G. Fouquier is now with eXenSa, Paris and J. Anquez with Theraclion, Paris.
} 
actual thickness (an approximate thickness is sufficient, since it will be prone to modifications during deformations).

WB MRI consists of an important amount of data and semi-automated segmentation methods are desirable to process them. However, only few works have been dedicated to this task since it is an emerging modality. Most approaches rely on low level methods and were developed for adults' images. For instance, T1-weighted MRI data are thresholded, and SAT is identified through region growing in the early work presented in [3]. A fuzzy c-means following intensity correction is used in 10 to identified AT and an active contour model allows to separate SAT and VAT (visceral adipose tissues) afterwards. Data acquired with the DIXON sequence are processed by means of thresholding, mathematical morphology tools and connected component extraction in [9]. The most sophisticated method is proposed in 6], where anatomical landmarks such as the spine are identified to constrain the segmentation of both SAT and VAT. However, this work only focuses on the abdominal region of the subject's anatomy. This approach has later been adapted to the case of children (5 years old) in [7] where the reduced amount of fat is challenging. Whole-body images are considered but only the abdominal region is segmented.

Here a patient-adaptive method for the WB SAT segmentation is proposed. Our contribution is to propose a method to segment SAT in the whole-body at once. Furthermore, the proposed approach does not require data homogenization thanks to the regularization. The study concerns images of children between 10 and 17 years old and the proposed method is therefore the first one dealing with $\mathrm{SAT}$ in WB children images.

\section{MRI Database}
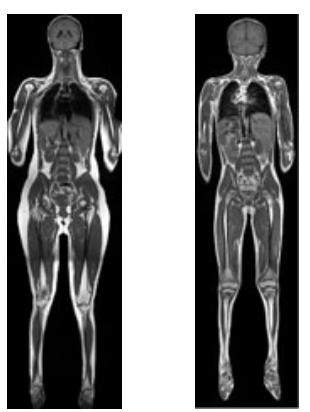

Fig. 1. Slices of WB images of two patients

WB MRI images of children have been acquired thanks to collaborating hospital, within protocols dedicated to the exploration of suspected autoinflammatory diseases such as chronic recurrent multifocal osteomyelitis (CRMO). These acquisitions are emerging thanks to shorter sequence durations. A set of 20 images has been acquired with the same Siemens scanner (1.5T) using $\mathrm{T} 1(T R=675 \mathrm{~ms}, \mathrm{TE}=11 \mathrm{~ms})$ sequence, using multiple coils. Examples of images are displayed in Figure 1. The table stops at each station. Depending on the patient, around 32 coronal slices are acquired with a slice thickness of $6 \mathrm{~mm}$. The reconstructed voxel size for all images is $1.3 \times 1.3 \times 7.2 \mathrm{~mm}^{3}$. The total scan time is $97 s$ for $T 1$ acquisitions. Due to the strong anisotropy, the data exhibit a lot of partial volume effect. Due to the use of multiple coils, images actually result from the composition of 4 or 5 images (depending on the patient height), and some artifacts may appear such as missing parts due to field size or lower intensity at the transition between two images. An example of the latter can be seen at the arms level in Figure 1 on the left. 


\section{SAT Segmentation}

The proposed method for SAT segmentation is based on a minimal surface approach. To make the method automatically adaptive to each patient, the grey level histogram is first analyzed to separate the background. Then a key feature of the method consists in using prior spatial information on the SAT location to define two regions, included in SAT and in the rest of the body, respectively: each voxel on the surface of the body is considered as fat and each voxel far enough from the surface is considered as belonging to the other class. A minimal surface approach is finally applied to estimate the best boundary between these two initial regions. Note that the hypothesis that the fat is the first tissue on the surface of the body is an approximation which is justified by the resolution of the images and their anisotropy, which make the skin not visible (its $1 \mathrm{~mm}$ thickness cannot be properly imaged with a voxel of $1.3 \times 1.3 \times 7.2 \mathrm{~mm}^{3}$ ).

\subsection{Identification of the Body Silhouette}

Figure 2(a) presents the histogram of the image displayed in Figure 1)(a). Similar histograms are observed on the whole database. Values corresponding to the background form a peak which decreases quickly. A second peak corresponds to the intensities of the muscles and the soft tissues (such as abdominal organs). The background mask is obtained by thresholding the original image. The selected threshold corresponds to the lowest value between the two identified peaks, and is determined automatically as the first valley of a filtered version of the histogram. The result (Figure 2(b)) separates the body from the

a)

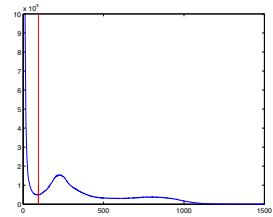

e)

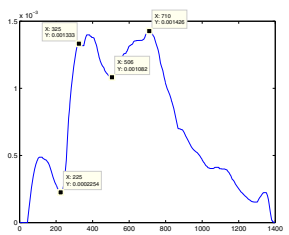

b)

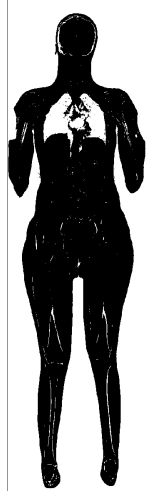

c)

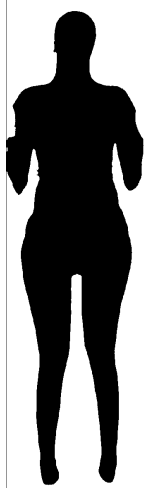

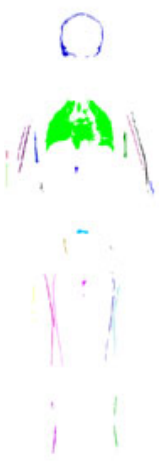

d)

Fig. 2. Automatic method to extract the body silhouette. The threshold is obtained from histogram analysis. (a) Histogram of a WB T1 MRI and threshold value (in red). (b) The thresholding result separates the background and some internal parts of the body, such as air-filled organs like lungs (in white). (c) Identification of the body silhouette. (d) The remaining dark components in the body. (e) Analysis of the silhouette of the body from the head to the feet: Each point represents the surface of the corresponding axial slice of the binary mask. The marked points correspond to the neck, the armpit, the waist and the hips. 
background, except components filled with air, liquid, or bones corticals (LCR, cortical bones, airways, lungs, part of the stomach, the heart and the intestine). A hole filling applied to the body allows us to add some of these components but a few others such as airways are directly connected to the background and thus cannot be filled. These components are disconnected using a morphological closing of the body component. A result is presented in Figure 2(c). The radius of the structuring element has been manually set and the same value is used for all images $(5 \mathrm{~mm})$. This operation adds unwanted parts in the mask such as fine space between the arms and the abdomen. Among the remaining components in the body (Figure 2(d)), the lungs may be identified as the biggest one (both lungs are generally connected in these images).

\subsection{Separation of the Main Parts of the Body}

As mentioned above, it is possible to consider that each voxel on the surface of the body belongs to SAT. To identify the body surface, the body mask alone is not sufficient since during the acquisitions the patient's hands lie on the thighs, the arms are sometimes in contact with the abdomen, and thigh fat may be in contact. Therefore, we propose to roughly separate the body into its main parts, namely head, thorax, abdomen, shoulders, arms, forearms, hips, thighs and legs. This identification allows us to cope with unwanted connections and to guarantee that different parts that should be separated are actually so.

The information on the image orientation is used to analyze the silhouette of the body, following a central axis from the top of the head to the feet. For each axial slice orthogonal to this axis, the area inside the body is computed. Figure 2e presents the curve of these values. Reference points are then identified: the first peak corresponds to the middle of the head, the first valley to the neck. The shoulders (second peak), the hips (highest remaining peak) and the waist (lowest valley between shoulder and hips) are also identified. Peaks and valleys are detected on a filtered version of the curve (using a simple median filter). The curve is similar for all images in our database, which allows us to automate this step for all images.

A first automatic body parts identification is achieved using these reference points and results in a labeling of the main body parts illustrated in Figure 3(b). The shoulders region labeling also uses the segmentation of the lungs and more local analysis of the body mask. The initial labeling is then manually corrected to separate the arms from the abdomen and the thighs (see Figure $3(\mathrm{c}, \mathrm{d})$ ). Other errors are corrected at the same time. This connection step is the only manual step in our approach.

\subsection{Initialization of SAT Segmentation}

The minimal surface approach used for the final segmentation is optimized using a graph-cut method, and is based on two hard constraining regions, one included 


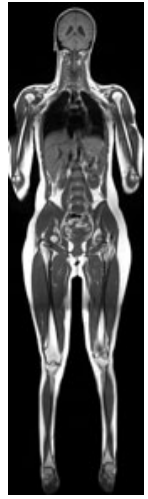

a)

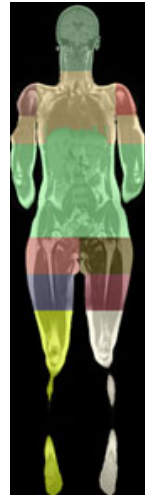

b)

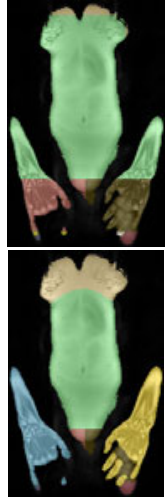

c)

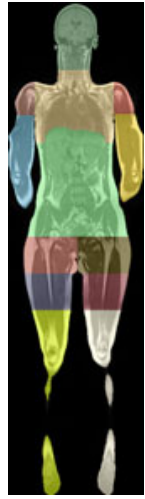

d)

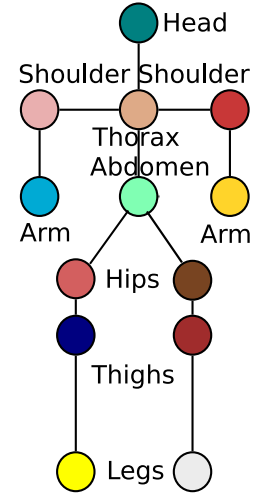

e)

Fig. 3. (a) A slice of an image. (b) Automatic labeling: the arms have the same label as the thorax and the abdomen. The hands have the labels of hips. (c) Separation of the hands from the thighs. (d) Corrected labeling. Each forearm (including the hand) has its own label. (e) Connections between body parts.

a)

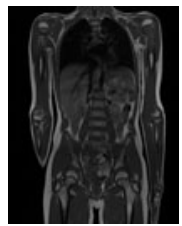

c)

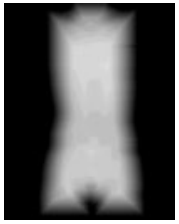

b)

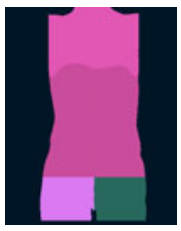

d)

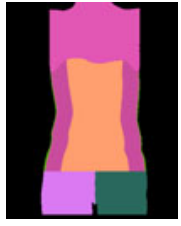

e)

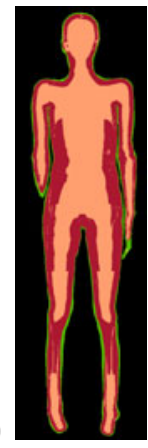

Fig. 4. Initialization derived from the labeling. Each voxel on the surface of the body is marked as SAT. Voxels far enough from the surface are marked as non-fat. (a) Here the arms are in contact with the abdomen. Each body part given by the previous step is processed independently. (b) The abdomen is merged with anatomically neighboring body parts (such as thorax or hips, but not arms). (c) Distance map from the background. (d) Extraction of border and inside classes for the selected body part. (e) Results from each part are merged to provide the initialization: each voxel in green belongs surely to SAT while each voxel in brown belongs to the inside class. The remaining voxels (in red) are not classified yet.

to a non-authorized label and thus the voxel is on the surface of the body. For example a voxel which is in the arm and neighbor of a background voxel is

in SAT and one included in non-fat regions, called "inside region". These two regions will be the seeds of the graph model and constitute the initialization of segmentation.

For SAT, each boundary voxel of the body is considered as part of it, as explained before. The previous body part identification allows us to detect the body surface by considering each part independently. For each voxel of the border of a region, there are three possibilities: (i) it has a neighbor in the background, (ii) it is connected to an authorized label and thus the voxel is considered inside the body, (iii) it is connected 
always on the surface of the body. If a voxel of the arm is neighbor of an abdomen voxel, then this voxel is also on the surface of the body. But if a voxel of the arm is neighbor of a shoulder voxel, then it is not on the surface of the body.

For the inside class, each region is considered again and merged with the neighbor regions having an authorized connection (as defined above). Figure 4(b) shows the merging of the abdomen and its neighbors (thorax and hips). A distance map to the background is computed (Figure 4(c)) and used to perform an erosion whose size depends on the body part: sequential erosions are performed with an unitary ball until a given ratio of the initial body part volume is kept. For elongated body parts such as legs, the structuring element is a disk in the axial plane and not a ball. The eroded region is then masked by the original region mask to provide the inside class (for the abdomen in our example). This is repeated for each body part. The obtained initialization is illustrated in Figure 4(e) where voxels in green belong surely to fat and voxels in light brown belong to the inside class. Voxels in red are not assigned yet to any class, and the aim of the segmentation will be to find the best segmentation surface within this red region.

\subsection{Segmentation by Minimal-Surface Approach}

The final segmentation is performed using a minimal-surface method, minimizing the following energy function: $E(l)=\sum_{p} D\left(p, l_{p}\right)+\beta \sum_{p, q} V(p, q)$ with $p$ and $q$ two points, $l_{p}$ a label, $D()$ a data fidelity term, $\beta$ a fixed coefficient and $V$ a regularization term. The data fidelity term is defined for each voxel except for the background using the a priori information previously defined. For each unclassified voxel, the probability for this voxel to belong to each class is given by a Gaussian distribution of the intensity of each class (muscles and tissues on the one hand, AT and bone narrow on the other hand) previously computed on the whole image. The minimal surface is computed generally between these two classes and the regularization allows us to separate SAT from other AT or bones.

The regularization term is defined as follows: $V(p, q)=\frac{1}{\max \left\{\left(1.0-\operatorname{grad}_{p q}\right), \varepsilon\right\}} \mid l_{p}-$ $l_{q} \mid$ with $\operatorname{grad}_{p q}$ an estimation of the normalized gradient between $p$ and $q$ which takes into account the image anisotropy. The parameter $\varepsilon$ is set to $10^{-6}$ in our experiments. The parameter $\beta$ is also fixed in our experiments and the same value is used for all images (0.75).

This energy is minimized using the graph-cut approach described in 1152 using the $\alpha$-expansion algorithm until convergence.

\section{Results and Discussion}

Experiments have been conducted on our MRI database. Figure 5 present results on three cases, where the manual segmentation appears in green, the SAT segmentation appears in blue, and 3D reconstructions of the two classes are shown as well. The first patient is a 10 year old girl, the second one a 13 year old 

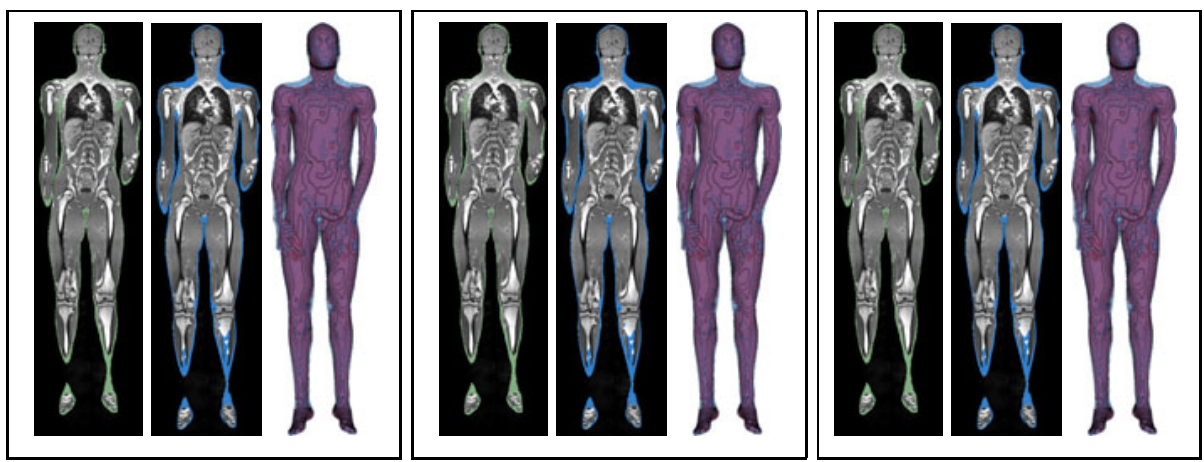

Fig. 5. Manual segmentation (in green), SAT segmentation (in blue): 10 year old girl (left) and overweighted 13 year old girl (right)

overweighted girl. The last one is a 17 year boy with very little fat. The results have been reviewed by radiologists, who confirmed their good quality, and the correct detection of all SAT locations. Moreover, the obtained results answer the needs raised by the foreseen applications: SAT is generally well recognized, it has the required topology (by construction, since the approach guarantees the topology of an empty sphere), and the fat thickness is approximately preserved. Some errors are inherent to the method: each voxel on the surface of the body is marked as fat, therefore small extremities such as fingers or ears are entirely considered as fat. Note that this will be corrected in a further step, since a skin layer will then be introduced on higher resolution reconstructions, before dosimetry simulations. Other errors are due to the poor resolution of the images in the coronal direction, making the transition between fat and bone sometimes unclear. Therefore some parts of the tibias are often marked as fat. Parts of the clavicles are also often classified as fat.

A quantitative evaluation of the segmentation was achieved thanks to 4 manual segmentations of SAT on the whole-body,

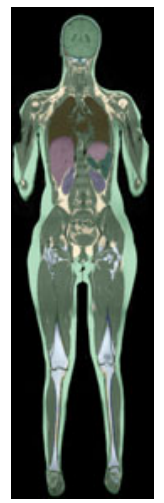

Fig. 6. 14 years old girl model including the 3 cases presented in Figure 5 . Each segmentation has been achieved by an expert user and takes approximately 4h per image. Manual segmentations have been reviewed by other radiologists. Table@presents the mean distance between the automated segmentation and the manual one for each of the 4 cases, as well as the similarity index. The best results are for the over-weight girl and the lowest for the skinny boy. The very low amount of fat in this case along with the partial volume effect make the manual segmentation challenging even for an expert. The resulting segmentation guarantees the topology, even if there is no fat to show. This may be a limitation of the method. When considering only the abdomen and the hips, results are better. The results on the 16 other images of our database show a similar quality, according to a visual inspection performed by medical experts. 
Table 1. Automated segmentation vs manual segmentation for 4 cases

\begin{tabular}{|l|cc|cc|cc|}
\hline & \multicolumn{2}{|c|}{ details } & \multicolumn{2}{c|}{ Whole Body SAT } & \multicolumn{2}{c|}{ Abdominal SAT } \\
\hline & Corpulence Age & Mean dist (vox) & Similarity & Mean Dist (vox) Similarity \\
\hline p1 & normal & 13 & 1.18 & $87.40 \%$ & 0.99 & $92.67 \%$ \\
p12 & over-weight & 13 & 1.28 & $88.11 \%$ & 1.06 & $92.71 \%$ \\
p15 & skinny & 17 & 1.94 & $65.44 \%$ & 1.22 & $77.42 \%$ \\
p16 & normal & 10 & 1.12 & $82.46 \%$ & 1.54 & $88.14 \%$ \\
\hline
\end{tabular}

These results are a useful first step towards the design of realistic WB models of children for numerical dosimetry simulations. Future work aims at segmenting more tissues and organs to complete these models. An illustration of one of these models is presented in Figure 6. Beside the primary goal of dosimetry simulations, interesting medical outcome could be derived from this segmentation. As pointed out in [4], the body fat distribution could be studied to analyze the body mass index and monitor therapy for obesity, or evaluate its change according to pathologies. Differentiating SAT from VAT is then important. Also body fat automatic segmentation allows fat distributions comparison in studies such as in 8 .

\section{References}

1. Boykov, Y., Kolmogorov, V.: An experimental comparison of min-cut/max-flow algorithms for energy minimization in vision. IEEE PAMI 26(9) (2004)

2. Boykov, Y., Veksler, O., Zabih, R.: Efficient approximate energy minimization via graph cuts. IEEE PAMI 20(12), 1222-1239 (2001)

3. Brennan, D., Whelan, P., Robinson, K., Ghita, O., O'Brien, J., Sadleir, R., Eustace, S.: Rapid automated measurement of body fat distribution from whole-body MRI. Am. J. of Roentgenology 185(2), 418-423 (2005)

4. Darge, K., Jaramillo, D., Siegel, M.: Whole-body MRI in children: current status and future applications. Eur. J. Radiol. 68(2), 289-298 (2008)

5. Kolmogorov, V., Zabih, R.: What energy functions can be minimized via graph cuts? IEEE PAMI 26(2), 147-159 (2004)

6. Kullberg, J., Johansson, L., Ahlström, H., Courivaud, F., Koken, P., Eggers, H., Börnert, P.: Automated assessment of whole-body adipose tissue depots from continuously moving bed MRI: A feasibility study. J. Magnetic Resonance Imaging 30(1), 185-193 (2009)

7. Kullberg, J., Karlsson, A.K., Stokland, E., Svensson, P.A., Dahlgren, J.: Adipose tissue distribution in children: Automated quantification using water and fat MRI. J. Magnetic Resonance Imaging 32(1), 204-210 (2010)

8. Machann, J., et al.: Standardized assessment of whole body adipose tissue topography by MRI. J. Magnetic Resonance Imaging 21(4), 455-462 (2005)

9. Martinez-Moller, et al.: Tissue classification as a potential approach for attenuation correction in whole-body PET/MRI: evaluation with PET/CT data. J. Nuclear Medicine 50(4), 520-526 (2009)

10. Sussman, D., Yao, J., Summers, R.: Automated measurement and segmentation of abdominal adipose tissue in MRI. In: IEEE ISBI, pp. 936-939. IEEE (2010) 